\title{
A molecular investigation into the genetic networks that respond to the mechanical characteristics of the microgravity environment experienced by $\boldsymbol{A}$. thaliana during spaceflight.
}

\author{
Jarret Henning, Richard Barker, Simon Gilroy \\ Botany Department; University of Wisconsin-Madison, Madison
}

\begin{abstract}
Plants respond to stresses related to microgravity. Calcium $\left(\mathrm{Ca}^{2+}\right)$ and Reactive Oxygen Species (ROS) have been shown to be involved in the signaling of rapid systemic response (figures 2 and 3; Gilroy et al., 2016 and Steinhorst et al., 2013). RNAseq has been used to identify genes affected by point mutations within the touch responsive gene $\mathrm{CML} 24$ (TCH2 in figure 1) identifying genes that are involved in the tch2 signaling pathway. By using bioinformatics, network analysis has been performed (figure 1) to help determine the role that the gene CP1 (AT5G49480.1) plays in touch response and calcium signaling. New phenotyping protocols have been developed to analyze the effect of mechanical stimulation through vibrations. This has produced a standardized way in which the effects of vibrations on plants are measured and tested, allowing us to determine whether the effects can be mitigated or enhanced through mutations. This work is funded by NASA and WSGC.
\end{abstract}

\section{Introduction}

Summary and objective: The Gilroy Lab TOAST database has identified a network of genes that respond to spaceflight and are important nodes in the terrestrial mechanical and pathogen response signaling pathways. The gene called Calcium binding Protein 1 (CP1, AT5G49480.1) was believed to play a role in the size of Arabidopsis thaliana plants as well as the underlying response mechanisms to abiotic stress that that these plants experience. The purpose of this project was to help determine the functionality of CP1 and its relation to the Touch Two (TCH2, AT5G37770.1), the Respiratory Burst Oxidase Homolog protein D (RBOHD, AT5G47910.1), and the Calcium-Dependent Protein Kinase 26 (CPK26, AT4G38230.2) genes the three of which were related in touch stimuli and the associated pathways that were activated by external stimuli. $t c h 2 \&$ rbohD mutants were recently grown on the ISS as part of the Gilroy Lab APEX5 project; this project allowed for the analysis of how their genetic networks adapt to the stresses of spaceflight. Dissecting how these genetic and proteomic interactions work on Earth such as those shown in figure 1 have provided insights into the impact that spaceflight has and will continue to have on plants.

Footnote: Thanks to the Wisconsin Space Grant Consortium (WSGC) and the National Aeronautics and Space Administration (NASA) for their financial contribution. This work would not have been possible without it. (Award number: UGR18_6.0) 


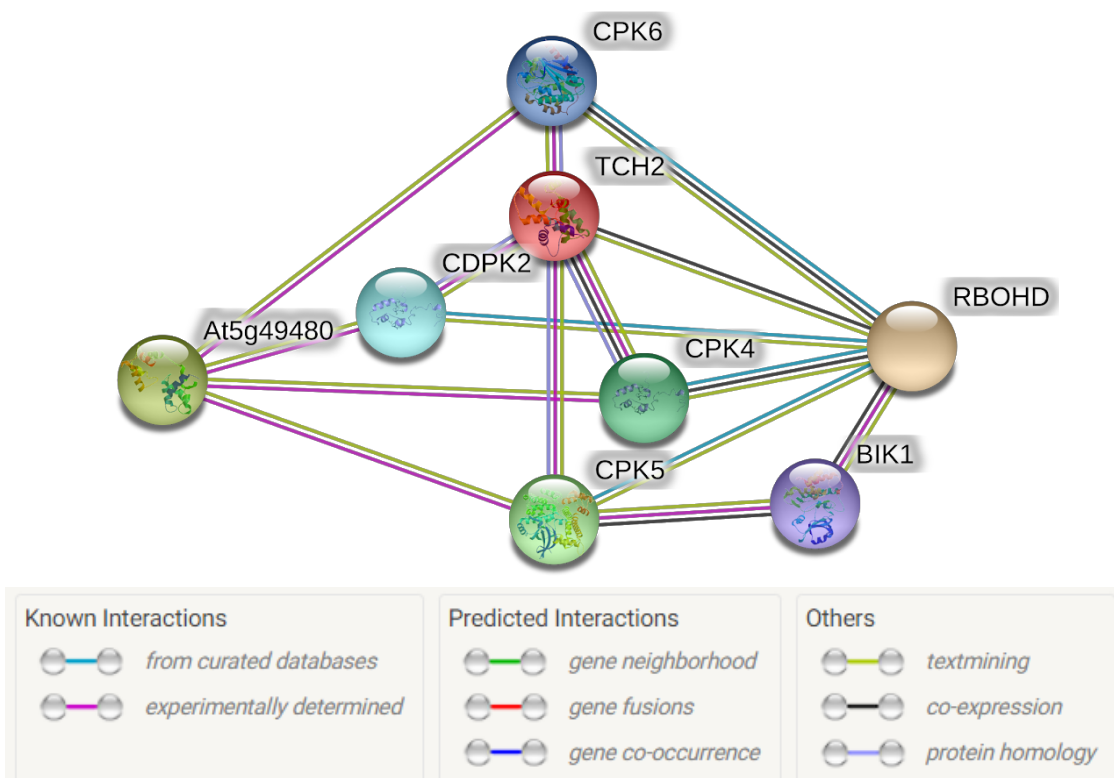

Figure 1: Network diagram of bioinformatically predicted interactions between CML24 and RBOHD were accessed from the String Database (https://string-db.org/) on the 2/2/2018.

Background: The calcium ROS wave was reviewed by Gilroy et al. (2016). In this paper, evidence was presented indicating that rapid systemic signaling was essential for plant acclimation to abiotic stress. The associated pathways have been explored to some degree about the function of the proteins involved. CP1 was experimentally linked to the same $\mathrm{Ca}^{2+}$ pathway as the ROS and calcium waves with a degree of confidence; however, the exact relationship and interactions of $C P 1$ have yet to be explored (figures 2 and 3). It has been important to understand this relationship due to the behavior of plants when exposed to abiotic stress such as those found in Arabidopsis. ROS and calcium waves have been seen to propagate through Arabidopsis as a result of abiotic stress and, as a way to acclimatize, have been seen to mediate systemic cell-tocell communication (Gilroy et al., 2016). In the Gilroy et al., (2016) paper and another paper by Steinhorst et al., (2013), it was shown that TCH2 and RBOHD were involved in the same $\mathrm{Ca}^{2+}$ signaling pathway as CP1 that corresponds to the calcium and ROS wave transmission throughout the plant. Figure 4 showed that the genes involved in response to ROS signaling were altered during spaceflight in the BRIC19 study by the Gilroy Lab. Due to this change observed in ROS response signaling, rbohD and cat-2 mutants were used in the vibration study to further understand how specific mutations within the ROS signaling pathway effect the root system architecture (RSA) of $A$. thaliana. During the Gilroy Lab's APEX5 mission, the RSA of $A$. thaliana was observed to change dependent on the microgravity environment experienced on the ISS (figure 5). This difference in RSA may have been due to hypoxia, gravity, or even vibrations from spaceflight (Cho et al., 2019). The idea of vibrations affecting plant growth in space was not investigated in as much depth as the other potential causes of growth differences, hence the decision to further study how vibrations may affect plant growth. 
(A)


Figure

2: Calcium-ROS waves are is used in $A$. thaliana stress response. RICR, ROS-induced $\mathrm{Ca}^{2+}$ release; $\mathrm{CICR} \mathrm{Ca}^{2+}-$ induced $\mathrm{Ca}^{2+}$ release; PM, plasma membrane; PD, plasmodesmata; CPK, Calcium-activated protein kinase.

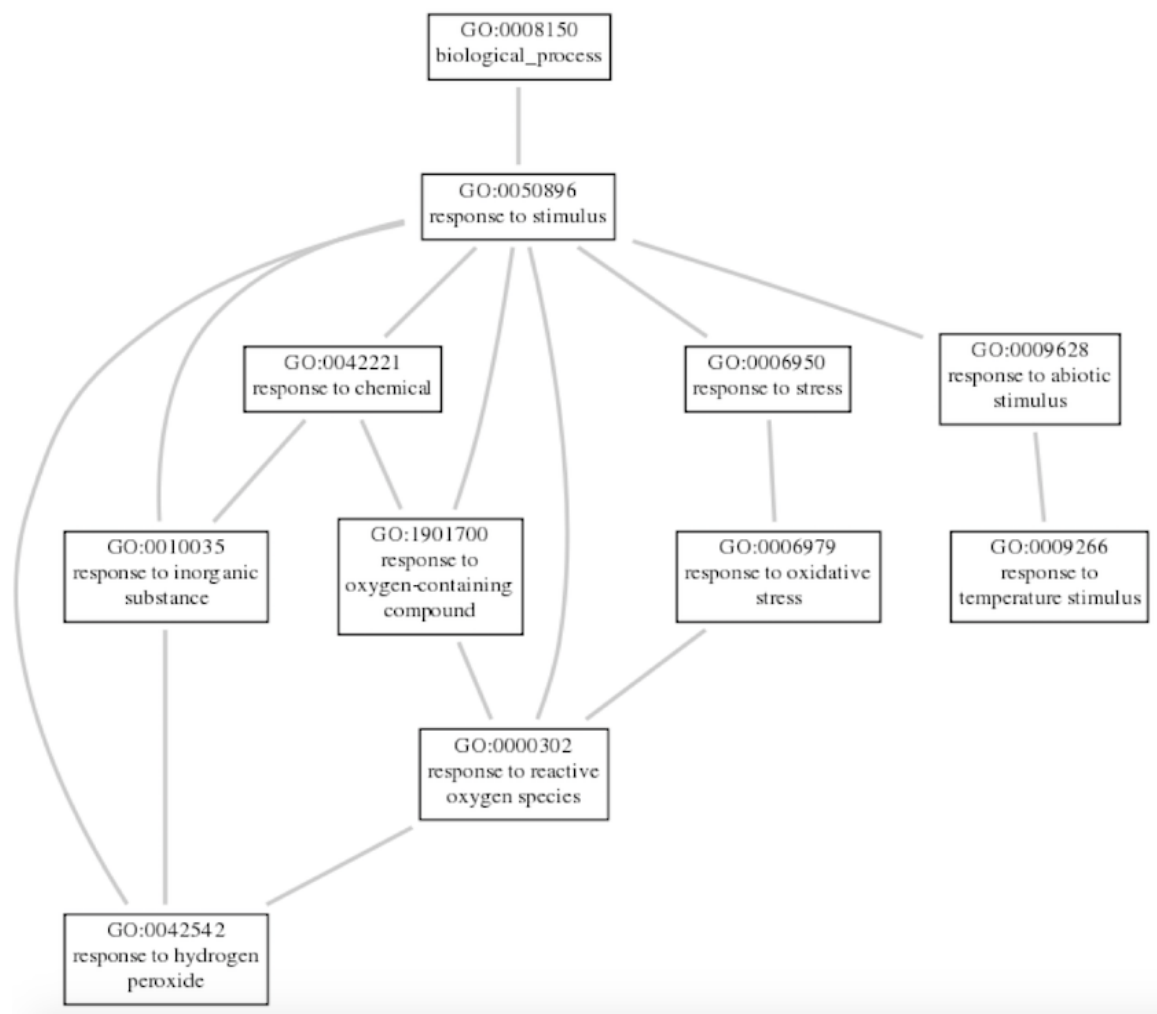

Figure 4: Genome Ontology analysis of RNAseq data generation by the Gilroy Lab BRIC19 study show that the genes involved in "response to ROS" were altered during spaceflight. 

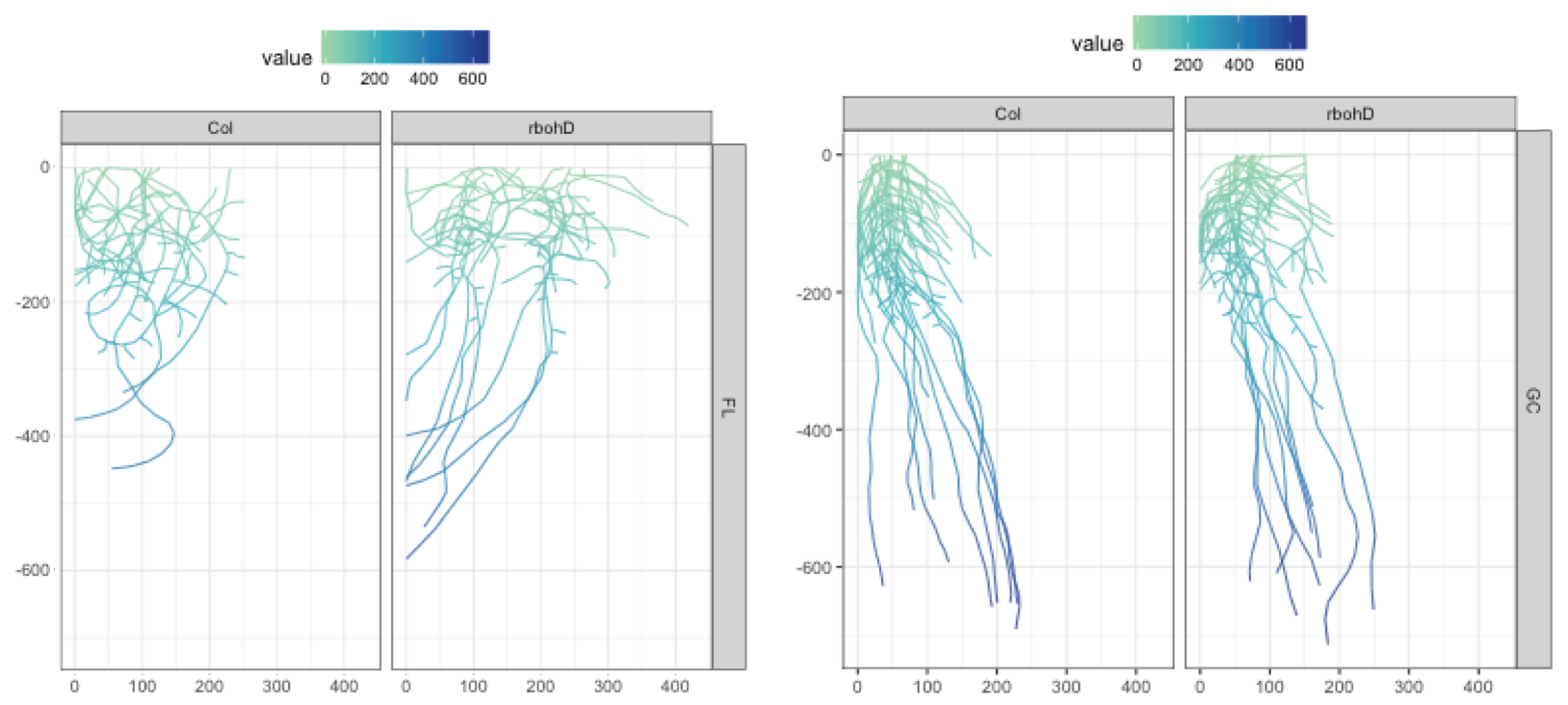

Figure 5: Comparison of the root systems from the APEX5 spaceflight experiment where the GC samples of Col-0 and $r b o h D$ were kept on the ground and the FL samples went aboard the ISS.

\section{Materials and Methods}

In this experiment, two different genotypes of $A$. thaliana, Ws- 2 and Col-0, and two mutants, cat-2 and rbohD, were observed. Each variant was observed in two different chambers; the control chamber and the "vibration chamber." Each variant had 11 seeds sowed onto 4 plates made of "Space Media," where space media is a $1 \%$ Phytogel, $0.24 \%$ ( $1 / 2$ strength) LS Nutrients, and $0.3 \%$ sucrose gel. These plates were wrapped in aluminum foil and then placed into a refrigerator for 24 hours so that the germination process between all of the variants would be consistent. After these plates were refrigerated, they were placed under white light for 48 hours and then were scanned into a computer so that the root system growth could be analyzed using ImageJ, RStudio, and R Shiny applications.

The two chambers were made from a 12x12x12 inch styrofoam cooler with 1 inch thick walls. In the bottom of the vibration chamber, a speaker was placed controlled by a Raspberry Pi. The control chamber did not have a speaker. In both chambers, a wooden platform was placed to raise all of the plates up to the same height. Over each chamber, a square piece of plexiglass was placed to allow light into each chamber.

Each variant was distributed onto four Space Media plates, two plates per variant were put in the vibration chamber, and the other two plates were placed in the control chamber. The speaker in the vibration chamber produced a $1000 \mathrm{~Hz}$ frequency for one second and then was silent for one second; over the course of 2 weeks, it repeated this pattern. Every 48 hours during this 2 week period, the plates were taken out of their chambers and scanned. Figure 6 is an example of the scans produced by this method. Each of these scans were split into individual photos by genotype, date, and chamber so that root growth analysis could be performed using ImageJ and the data were analyzed using RStudio (figure 7). 
Figure 6:

On the left

is an image of the plates $1,3,5,7$, and 9 from the control chamber and on the right is an image of the correspondi ng plates from the vibration chamber. Resolution 300 dpi.

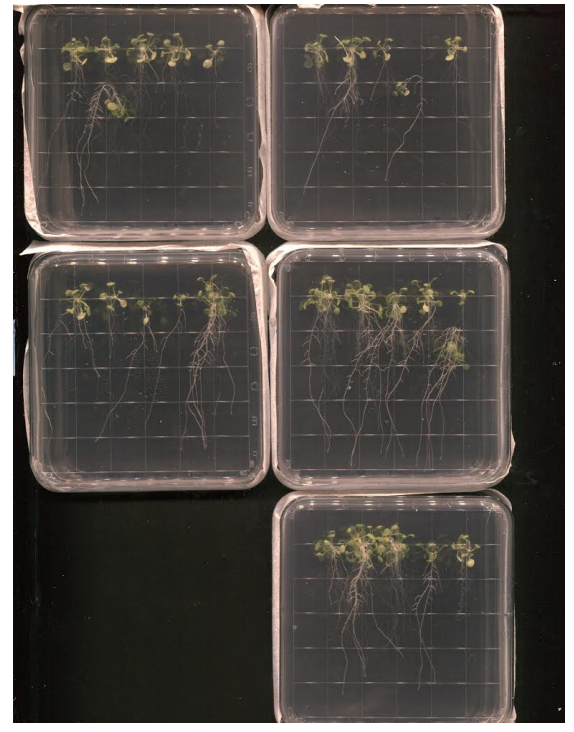

100200300400500

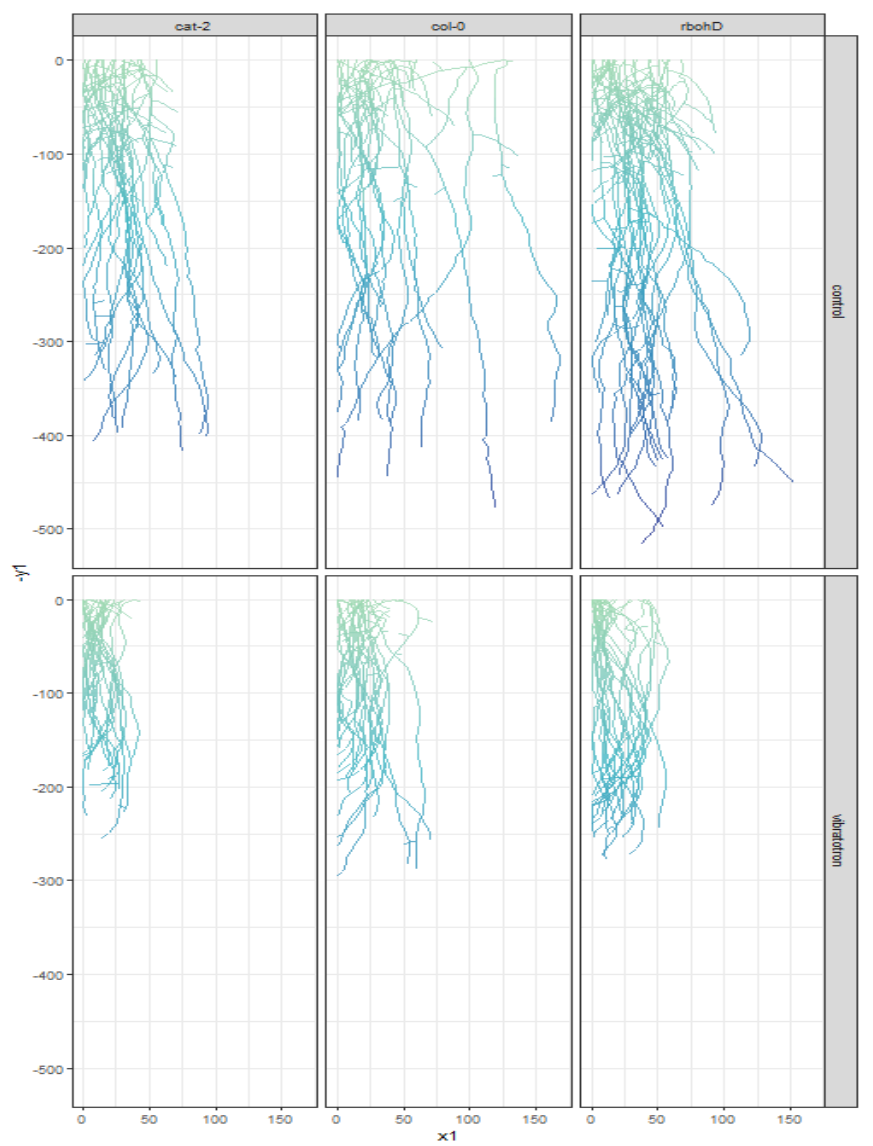



Figure 7: A comparison of the root systems of wild type, cat-2 and rbohD mutants in the vibration $(1000 \mathrm{~Hz})$ and control chambers. 


\section{Results}

Figure 7 shows that the $A$. thaliana variants in the vibration chamber had a difference in root growth rates and the direction in which the plants grew. The roots were shorter and generally grew in a straight path in the vibration chamber and they were less consistent in the control chamber. This can be seen by looking at how much or how many roots deviate from a main path in figure 7. The rbohD and Col-0 variants tended to skew more in the control chamber and in the vibration chamber than the cat-2 variant. Though, in the control chamber, the roots did not always grow similarly and this can be seen by how spread out the root system architecture is in figure 7 .

Figure 8 shows a statistical analysis of the impact on total root system length as well as root system growth in A. thaliana. Figure 8. A shows that at time $=3$ days, which was day 5 of the experiment, there was a statistical significance in the total root system length within the vibration chamber (vibratotron in the figures) and between the vibration chamber and the control chamber. It also shows that at time $=3$ the rate of root system growth, which is the change in root length per day, is much higher in the control group than in the vibration chamber. rbohD was also seen to be more resistant to the vibrations than the other variants within this experiment.
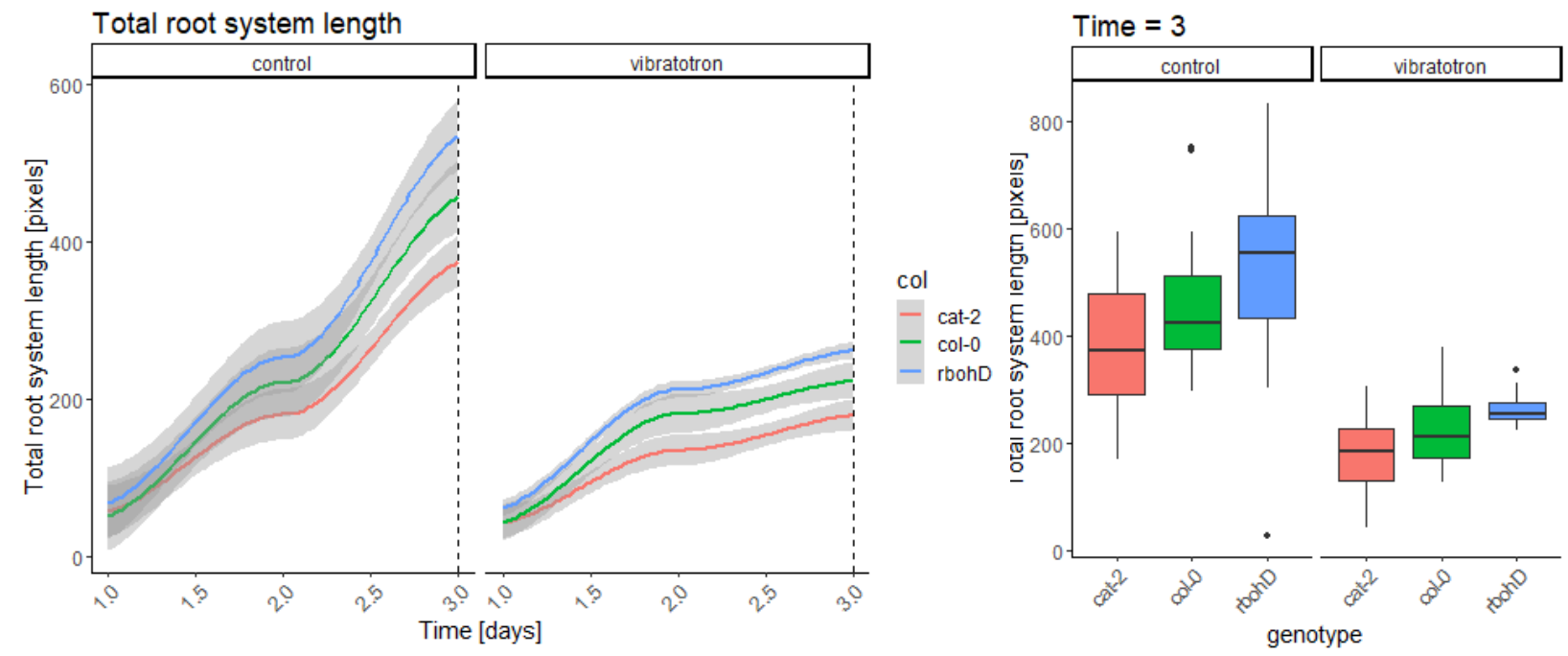

Figure 8: Impact on total root system length as well as root system growth in A. thaliana. (A: Above) Time course, (B: Right) Quantification at 3 days at $1000 \mathrm{~Hz}$. Note the rbohD variants are more resistant to vibrations. Vibratotron is the same as the vibration chamber. 


\section{Discussion}

Root growth rate and direction in A. thaliana can be altered by vibrations and this may happen because vibrations are treated as mechanical stress and touch stimuli. The rbohD variants have been shown to be more resistant to touch stimuli and this is further shown in figure 8 . This figure shows that the $r b o h D$ variants were more resistant to the vibrations produced in the vibration chamber, which suggests that sound waves, or vibrations, are treated similarly to touch stimuli by $A$. thaliana using $\mathrm{Ca}^{2+}$ and ROS waves. This knowledge can be used in the future to develop test standards for measuring the effect that vibrations have on root system growth.

\section{Future Work}

Due to time constraints, we were unable to create $C P 1$ variants such as T-DNA insertional mutants or EMS included SNPs. To address this, future work may include the creation of $C P 1$ knockout mutants and/or overexpression lines to further understand the role that CP1 plays in the $\mathrm{Ca}^{2+}$ signaling and touch sensing pathways. In addition to creating new genotypes with potentially altered sensitivity to vibrations, we also plan to test a range of various frequencies $(\mathrm{Hz})$ of vibrations to see if it would be possible to direct or even promote root growth. The long term goal of this research is to investigate if it is possible to direct plant root growth in the absence of gravity using audio stimuli alone.

\section{Microgravity research campaign}

\section{Access to Microgravity}

1. Drop towers

2. Parabolic flights alternations between hypergravity and microgravity for short periods of time $(<1 \mathrm{~min})$

3. Blue Origins 4 minutes of microgravity

4. Growth on the international space station

5. Bion satellite

\section{Potential plant growth hardware}

1. BRIC \& BRIC LED to small to insert speakers

2. VEGGIE growth chamber

3. Advanced plant habitat

Based on the methods demonstrated during this study, access to microgravity for relatively long periods of time is required to test if audio vibrations can be used to direct root growth in the absence of gravity. We postulate that microgravity opportunities offered by drop towers, parabolic flights, and Blue Origin's NewShepard flights would be capable of testing this theory. Thus, either access to a Bion satellite flight or access to astronaut time on the international space station are the only platforms that offer $>5-8$ days growth required. Due to the need to create an audio vibration it is unlikely that the BRIC (Biological Research in Canister) system would be a viable growth environment. So, we conclude that either the Advanced Plant Habitat or the VEGGIE growth systems (Made by Orbitec / Sierra Nevada Corp, Middleton, Wi) would both provide ideal environments to test the potential use of audio vibrations to direct root growth in 
microgravity. However, this would require the development of a smaller custom built audio/ vibration source that would require NASA certification for flight.

\section{Limitations within the study}

Although the Ws-2 ecotype was included to assess the potential natural variation that may exist with respect to vibrational stimuli, these data were not presented as these samples became contaminated during the experiment.

\section{Acknowledgements}

I would like to thank Dr. Richard Barker, Professor Simon Gilroy, and Dr. Sarah Swanson for inspiring me to take on this challenge and help promote scientific research in the field of Astrobotany. I would also like to personally thank Professor Simon Gilroy for allowing me to work in his lab and give me the wonderful opportunity to conduct my own research; Dr. Richard Barker for helping me figure out how I was going to conduct the research and analysis; and Dr. Sarah Swanson for critical discussion of the BRIC19 and APEX5 spaceflight methodology.

\section{References}

Cho, W. G., Barker, R. J., Kim, S. H., Swanson, S. J., Gilroy, S., (2019, January 15). Variation in the transcriptome of different ecotypes of Arabidopsis thaliana reveals signatures of oxidative stress in plant responses to spaceflight. Retrieved January 20, 2019, from https://www.ncbi.nlm.nih.gov/pubmed/30644539

Gilroy, S., Białasek, M., Suzuki, N., Górecka, M., Devireddy, A. R., Karpiński, S., \& Mittler, R. (2016, July 01). ROS, Calcium, and Electric Signals: Key Mediators of Rapid Systemic Signaling in Plants. Retrieved August 08, 2018, from http://www.plantphysiol.org/content/171/3/1606

Steinhorst, L., \& Kudla, J. (2013, October 01). Calcium and Reactive Oxygen Species Rule the Waves of Signaling. Retrieved August 08, 2018, from http://www.plantphysiol.org/content/163/2/471 